\title{
Fuzzy controller optimization using a genetic algorithm for non-collocated semi-active MR based control of a three-DOF framed struture
}

\author{
Manuel T. Braz-César \\ Dept. of Applied Mechanics \\ Polytechnic Institute of Bragança \\ Bragança, Portugal \\ brazcesar@ipb.pt
}

Rui C. Barros

Dept. of Civil Engineering

Faculty of Engineering of the University of Porto

Porto, Portugal

$\underline{\text { rcb@fe.up.pt }}$

\author{
Pedro L.P. Folhento \\ MSc. in Civil Engineering \\ Polytechnic Institute of Bragança \\ Bragança, Portugal \\ pedro.lp.folhento@gmail.com
}

\begin{abstract}
This paper aims to explore the usefulness of a simple genetic algorithm (GA) optimized Fuzzy Logic Controller (FLC) to reduce the response of a three-DOF framed structure equipped with a MagnetoRheological (MR) damper. These actuators can be controlled in bi-state control mode and/or in a semi-active configuration by continuously adjusting the amount of damping according to the actual response. Generally, model based controllers are designed to determine the actuator output. In recent years, soft computing techniques have been implemented to deal with the highly non-linear nature of structural systems. Among others, fuzzy based controllers seem to be adequate approach for these cases due to the inherent ability to deal with uncertain systems. However, a FLC design requires a wide experience in operating the system. This can be very difficult to implement in complex systems and several optimization techniques have been suggested to enhance the design process of fuzzy controllers. In this paper, a genetic algorithm (GA) optimized semi-active fuzzy based controller is proposed to reduce the seismic response of a three degree-offreedom (DOF) structure using a MR damper at the first DOF. The uncontrolled and controlled structural responses are compared to evaluate the effectiveness of the semi-active fuzzy based controller.
\end{abstract}

Keywords- Structural control; Fuzzy logic; Genetic Algorithms; Semi-active system; MR Dampers..

\section{INTRODUCTION}

Conventional controllers are usually based on mathematical models which describe the dynamic behavior of the system. Thus, a precise formulation of the process is required to obtain a reliable control action. Fuzzy logic systems are able to include human expert knowledge to control complex systems that cannot easily be incorporated in the mathematical model by translating the linguistic control rules into a fuzzy set theory (Jang, 1993). Then, fuzzy controllers do not require detailed information of the system enhancing the robustness of control systems to non-linear or uncertain variables. However, defining fuzzy parameters is a time consuming task, usually carried out with an experience-based trial-and-error procedure resulting in non-optimal solutions. Soft computing techniques such as neural networks and Genetic Algorithms (GA) have been proposed to provide a more efficient approach to design fuzzy controllers (Byrne 2003, Ali \& Ramaswamy, 2008).

Genetic algorithms are computational search techniques that simulate natural genetics and biological evolution. They provide an effective approach to optimize fuzzy parameters, either membership functions (MFs) or rule sets or both at the same time. Besides, the optimization procedure can also be designed to search for optimal gain or scaling factors which are of much importance to improve the control performance and reduce stability issues.

In this paper, an optimized fuzzy logic based controller is presented for the control of the damping states of a MagnetoRheological (MR) damper aimed to reduce the response of a three degree-of-freedom (DOF) structural system. These semi-active actuators can be controlled in passive control mode with constant damping ratios or in a semi-active configuration by continuously adjusting the amount of damping according to the actual response.

The fuzzy controller is used to compute the control signal (operating current) based on the measured velocities of the first and third masses (i.e., using velocity feedback).

A genetic algorithm optimization approach is applied to determine the optimum parameters (gains or scaling factors) and reshape the membership functions of the fuzzy controller in order to satisfy the required control signal while minimizing the system response. The simulation results of the investigated control system are provided and discussed. 


\section{NUMERICAL MODEL}

The mechanical discrete model of the 3-DOFs system is displayed in Fig. 1. The structure represents of a 3-story building under seismic excitation with a semi-active MR actuator located between the ground and the first floor.

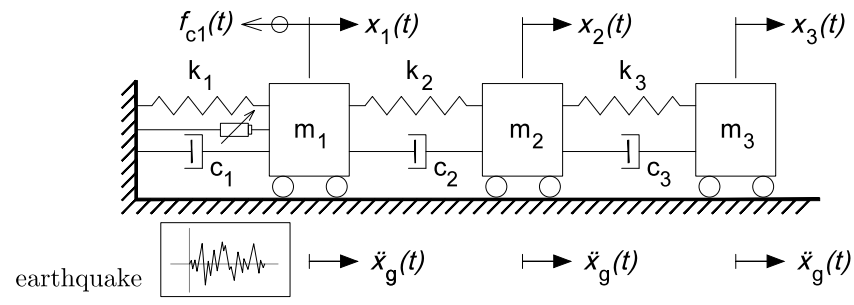

Fig. 1. Discrete model of the 3-DOFs system under seismic excitation.

The equation of motion describing the response of the system is defined as

$$
\mathbf{M}^{*} \quad(t)=\boldsymbol{\Gamma} f_{c 1}(t)-\mathbf{M} \lambda^{*} \cdot
$$

where $\mathbf{X}(t)$ defines the displacement response (mass displacement), $\mathrm{f}_{\mathrm{c} 1}(t)$ the control force, $\ddot{\mathrm{x}}_{\mathrm{g}}(t)$ the ground acceleration, $\mathbf{M}_{(3 \times 3)}, \mathbf{C}_{(3 \times 3)}$ and $\mathbf{K}_{(3 \times 3)}$ are the mass, damping and stiffness matrices, respectively, given by

$$
\begin{gathered}
\mathbf{M}=\left[\begin{array}{ccc}
m_{1} & 0 & 0 \\
0 & m_{2} & 0 \\
0 & 0 & m_{3}
\end{array}\right] \\
\mathbf{C}=\left[\begin{array}{ccc}
c_{1}+c_{2} & -c_{2} & 0 \\
-c_{2} & c_{2}+c_{3} & -c_{3} \\
0 & -c_{3} & c_{3}
\end{array}\right] \\
\mathbf{K}=\left[\begin{array}{ccc}
k_{1}+k_{2} & -k_{2} & 0 \\
-k_{2} & k_{2}+k_{3} & -k_{3} \\
0 & -k_{3} & k_{3}
\end{array}\right]
\end{gathered}
$$

and finally $\boldsymbol{\Gamma}$ and $\lambda$ are location vectors of the control forces and the earthquake excitation, respectively given by

$$
\boldsymbol{\Gamma}=[-1,0,0]^{T} ; \quad \lambda=[1,1,1]^{T}
$$

The mass, damping and stiffness are defined as: $m_{1}=m_{2}=m_{3}=100 \mathrm{~kg} ; \quad c_{1}=175 \mathrm{Ns} / \mathrm{m}, \quad c_{2}=c_{3}=50 \mathrm{Ns} / \mathrm{m}$ and $k_{1}=k_{2}=k_{3}=6 \times 10^{5} \mathrm{~N} / \mathrm{m}$.

The seismic acceleration time history of the 1940 NS component of the El-Centro earthquake is used as the ground motion (see Fig. 2). A time-scaled seismic record (1:5) was used to ensure a ground motion compatible with the scale of the structural model.

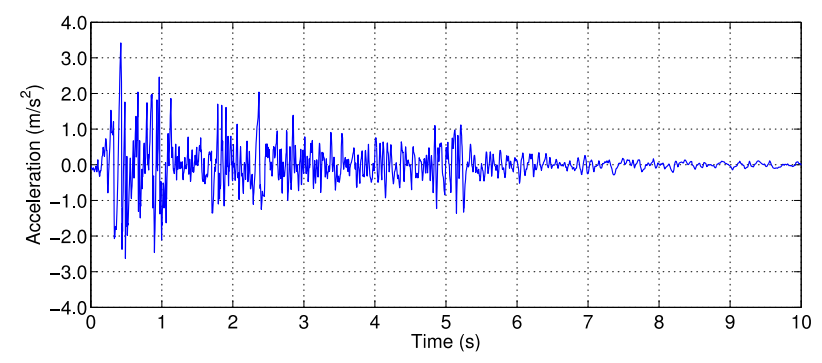

Fig. 2. N-S component of El-Centro earthquake (time scale of 1:5).

MR dampers are semi-active devices whose damping properties can be modified in real time by changing the magnetic field applied to the MR fluid inside the tube housing. This can be achieved by using a small external power source to control an electromagnet located in the piston rod to change the rheological properties (namely the yield stress) of the MR fluid. This leads to a strong modification in the damping state, which has proved to be notoriously difficult to accurately simulate in numerical models due to the highly non-linear and current dependent behavior of the actuator. Thus, several numerical models have been proposed to characterize the hysteretic behavior of MR dampers. The so-called modified Bouc-Wen model depicted in Fig. 3 seems to be particularly appropriate to simulate the dynamics of these actuators (Spencer et al. 1997).

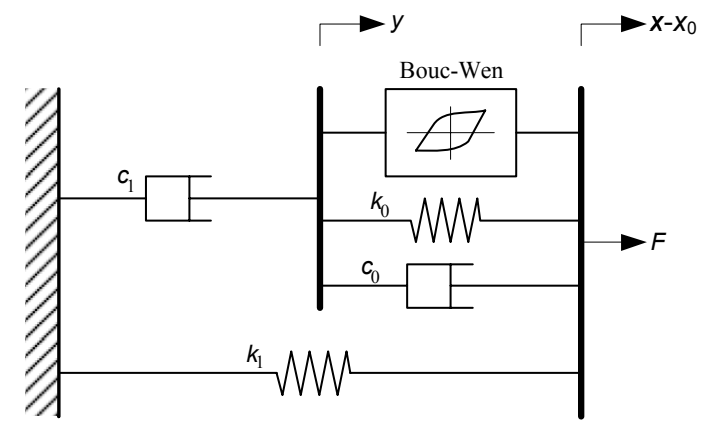

Fig. 3. Modified Bouc-Wen model for MR dampers (Spencer et al. 1997).

According with the parametric model, the damping force is described by the following equations

$$
F(t)=c_{1} \dot{y}(t)+k_{1}\left[x(t)-x_{0}(t)\right]
$$

$$
\dot{y}(t)=\frac{1}{c_{0}+c_{1}}\left(\alpha z(t)+c_{0} \dot{x}(t)+k_{0}[x(t)-y(t)]\right)
$$

in which $z(t)$ is the evolutionary variable obtained from

$$
\dot{z}(t)=-\beta|\dot{x}(t)| z(t)|z(t)|^{n-1}-\dot{\gamma} \dot{x}(t)\left|z(t)^{n}\right|+A \dot{x}(t)
$$

The model parameters are obtained from experimental analysis. It was found that some parameters are current/voltage independent, while other parameters are strongly current dependent since they are significantly affected by the operating current of the electromagnet (Braz-César \& Barros, 2012). 
In this study, the model parameters were defined based on the experimental investigation of a commercial MR damper model RD-1005-3 (Lord Corporation, USA). Thus, the current (or voltage) independent parameters are defined as: $\mathrm{A}=10.013$, $\beta=3.044 \mathrm{~mm}^{-1}, \gamma=0.103 \mathrm{~mm}^{-1}, \mathrm{k} 0=1.121 \mathrm{~N} / \mathrm{mm}, \mathrm{f}_{0}=40 \mathrm{~N}$ and $\mathrm{n}=2$. The current dependent parameters can be described by the following expressions

$$
\begin{aligned}
& \alpha(i)=\alpha_{3} i^{3}+\alpha_{2} i^{2}+\alpha_{1} i+\alpha_{0} \\
& c_{0}(i)=c_{03} i^{3}+c_{02} i^{2}+c_{01} i+c_{0} \\
& c_{1}(i)=c_{13} i^{3}+c_{12} i^{2}+c_{11} i+c_{1}
\end{aligned}
$$

In this case each coefficient is given by: $\alpha_{0}=38.2, \alpha_{1}=421.5$, $\alpha_{2}=905.1, \alpha_{3}=-826.7 ; \mathrm{c}_{0}=0.6, \mathrm{c}_{01}=11.0, \mathrm{c}_{02}=10.5, \mathrm{c}_{03}=-11.7$, $\mathrm{c}_{1}=4.7, \mathrm{c}_{11}=64.6, \mathrm{c}_{12}=57.0, \mathrm{c}_{13}=-54.4$.

A first-order filter involved in the current driver during a bang-bang (step command) signal is also included in the numerical mode defined by the time parameter $\eta=130 \mathrm{sec}^{-1}$.

\section{GA-OPTIMIZED FUZZY SYSTEM}

In the present case it is possible to establish a predictable pattern in the fuzzy reasoning engine based on the previous fuzzy surfaces, i.e., the control action should increase when the structural responses increase whether they have negative or positive signs. This entails a symmetric structure of the rule base, which can be exploited to simplify the optimization of the fuzzy controller. With this in mind, a genetic algorithm is used to design optimize the fuzzy controller. The proposed GA-optimization procedure is used to optimize the shape of the membership functions and also the fuzzification parameters $\mathrm{K}_{\mathrm{v} 1}$ and $\mathrm{K}_{\mathrm{v} 3}$ of the fuzzy controller (i.e., to perform a genetic tuning of the data base comprising the scaling gains and MFs). The defuzification gain has a constant value defined as $\mathrm{K}_{\mathrm{c}}=2.0$. To simplify the optimization procedure, the GA was developed based on the following assumptions:

- The universe of discourse of the input variables is normalized;

- The fuzzy surface will have a symmetric point at zero input signals;

- The input and output MFs will have the same shape.

Each input and output fuzzy variable is represented by seven triangular membership functions equally distributed along the universe of discourse. The linguistic variables are labeled as $\{\mathrm{NL}, \mathrm{NM}, \mathrm{NS}, \mathrm{ZE}, \mathrm{PS}, \mathrm{PM}, \mathrm{PL}\}$, where NL is Negative Large, NM is Negative Medium, NS is Negative Small, ZE is Zero, PS is Positive Small, PM is Positive Medium and PL is Positive Large. The expected range of the control signal $(0.0 \mathrm{~A}-0.5 \mathrm{~A})$ was used to define the universe of discourse of the output variable (Braz-César \& Barros 2017).

In this case, the mean max or middle of maxima (MOM) method was used for the defuzzification process to ensure that the maximum membership related with the maximum control action is a plateau. The noticeable symmetry of the fuzzy inference mechanism is used to set the inference rule matrix.
TABLE I. RULE-BASE MATRIX FOR TUNNING THE FUZZY CONTROLLER.

\begin{tabular}{c|c|c|c|c|c|c|c|c}
\hline \multirow{2}{*}{ Inputs } & \multicolumn{7}{|c}{ st $^{\text {stoor velocity }}$} \\
\cline { 2 - 9 } & NL & NM & NS & ZE & PS & PM & PL \\
\hline \multirow{4}{*}{} & NL & NL & NL & NL & NL & NM & NS & ZE \\
\cline { 2 - 8 } & NM & NL & NL & NM & NM & NS & ZE & PS \\
\cline { 2 - 9 } & NS & NL & NM & NS & NS & ZE & PS & PM \\
\cline { 2 - 9 } & ZE & NL & NM & NS & ZE & PS & PM & PL \\
\cline { 2 - 8 } & PS & NM & NS & ZE & PS & PS & PM & PL \\
\cline { 2 - 9 } & PM & NS & ZE & PS & PM & PM & PL & PL \\
\cline { 2 - 8 } & PL & ZE & PS & PM & PL & PL & PL & PL \\
\hline \multirow{2}{*}{}
\end{tabular}

The symmetric rule-base matrix used for tuning the proposed fuzzy controller produces a fuzzy inference system with 49 rules (Table I). The GA was coded to find a solution using a random population size of 50 individuals after 100 generations with a crossover probability of 0.60 . Every individual consist of 11 variables coded in 10 bit binary form. The fitness function to be minimized is the sum squared error (SSE) performance criterion defined as

$$
J_{S S E}=\sum_{j=1}^{n}\left(\hat{y}_{j}-y_{j}\right)^{2}=\sum_{j=1}^{n} e_{j}^{2}
$$

where $e_{j}=\left(\hat{y}_{j}-y_{j}\right)$ is the error between the desired output $y_{j}$ and the predictive value $\hat{y}_{j}$ estimated by the GA. A flowchart of the GA-optimization procedure is shown in Fig. 4.

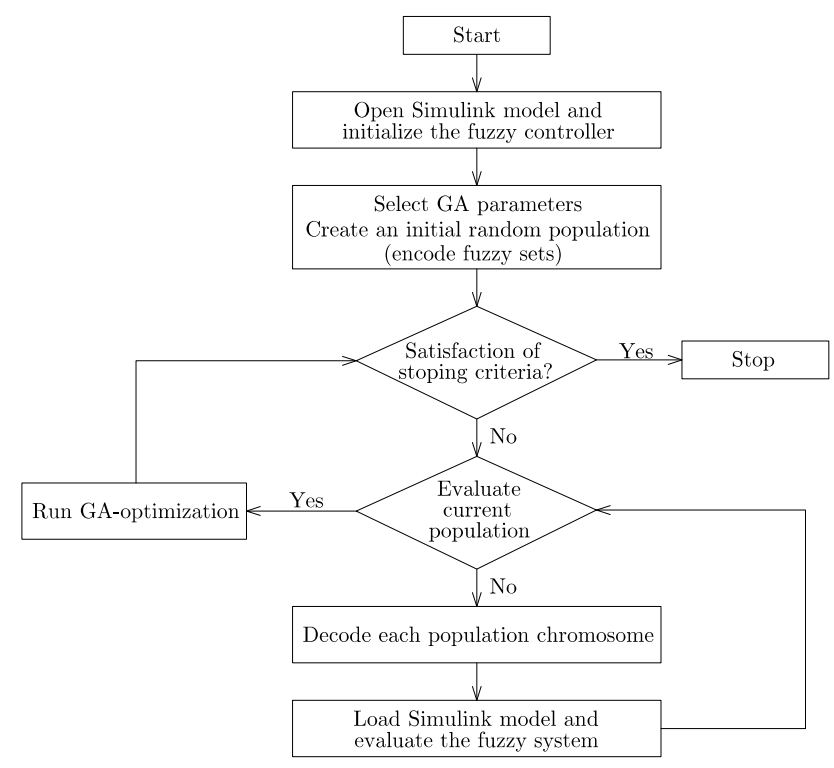

Fig. 4. Flowchart of the GA-optimization procedure

A numerical analysis was carried out to determine the fuzzy system that provides the best solution. The proposed fuzzy logic control system is derived from a Linear Quadratic Regulator (LQR) controller. The data obtained from the optimal controller is used as a reference to train a fuzzy based controller via a Genetic Algorithm optimization procedure. The resultant fuzzy surface is shown in Fig. 5. 

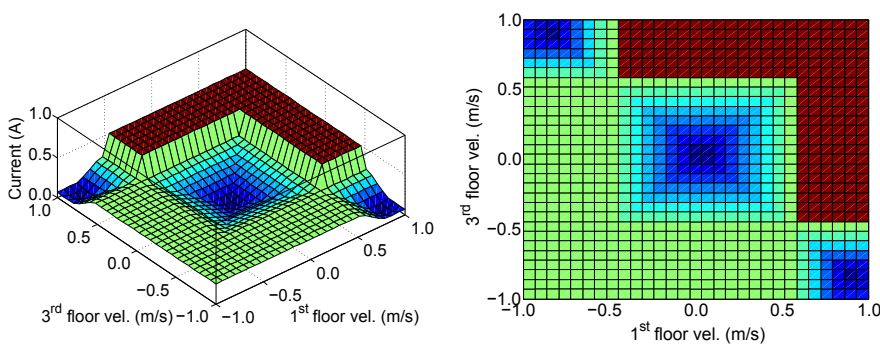

Fig. 5. Surface plots of the GA optimized fuzzy inference system.

The stair-step profile results from the defuzzification method used to obtain the output signal and also the constraints imposed on the membership functions. The resultant fuzzy surface has a symmetric axis along the diagonal line joining the two points of the boundary edge defined by the maximum positive value of the normalized universe of discourse, which is in accordance with the assumptions prescribed to define the rule-base. The controller computes a mid-range control signal when both velocities have negative values, which is related with the specificity of this structural system. The optimal values of the scaling factors for each input variable were also determined during the optimization procedure. The fuzzification factors that normalize the first and third floor velocities to $[-1,1]$ are defined as $\mathrm{K}_{\mathrm{v} 1}=3.91$ and $\mathrm{K}_{\mathrm{v} 2}=5.22$, respectively.

The maximum control output is achieved when both floor velocities are large and have the same signs. The fuzzy controller delivers the lowest control signal when both velocities are large but have opposite signs. A small control signal is also required when both input variables are around their null value (small velocities). The controller delivers a zero output value when both input variables have null values.

\section{NUMERICAL SIMULATION}

A numerical model was developed to investigate the performance of the fuzzy controller in reducing the response of the 3-DOFs system. The Simulink model of the proposed semi-active fuzzy controller is shown in Fig. 6. As can be seen, the FLC was designed using only the first and third floor velocity measurements to compute the required control signal.

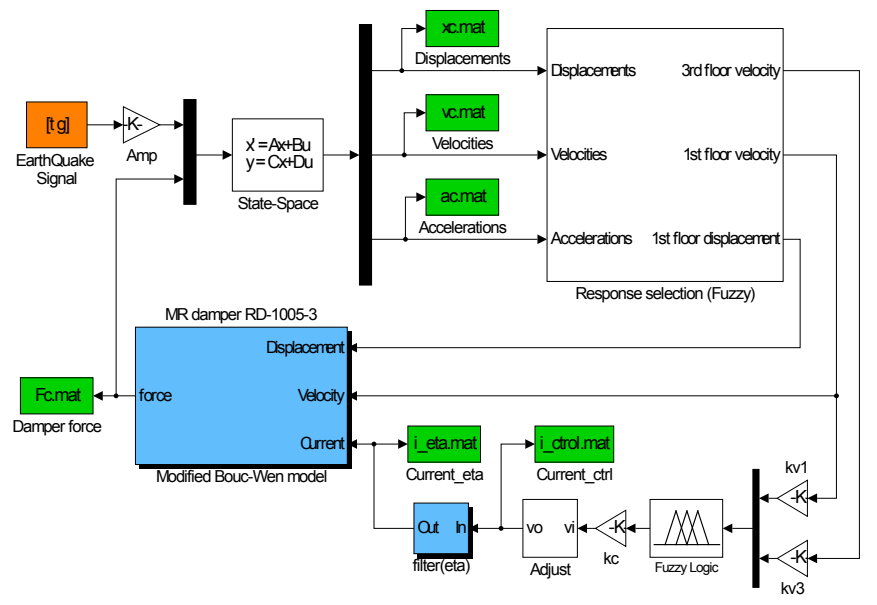

Fig. 6. Simulink model of the proposed semi-active FLC.
The uncontrolled response is compared with passive and semi-active controlled responses in order to assess the effectiveness of the proposed GA-optimized fuzzy based controller. Passive control modes represent the off-damping and on-damping states related with low and high damping forces respectively. The results are summarized in Table II.

TABLE II. PEAK RESPONSES (NS EL-CENTRO RECORD, TIME SCALE 1:5).

\begin{tabular}{|c|c|c|c|c|c|c|}
\hline \multicolumn{2}{|c|}{ Control strategy } & $\begin{array}{c}\mathrm{X} \\
(\mathrm{cm})\end{array}$ & $\begin{array}{c}\dot{\mathrm{X}} \\
(\mathrm{cm} / \mathrm{s})\end{array}$ & $\begin{array}{c}\ddot{\mathrm{X}} \\
\left(\mathrm{cm} / \mathrm{s}^{2}\right)\end{array}$ & $\begin{array}{l}\text { Drift } \\
(\mathrm{cm})\end{array}$ & $f(\mathrm{~N})$ \\
\hline \multirow{3}{*}{\multicolumn{2}{|c|}{ Uncontrolled }} & 0.695 & 27.09 & 1305 & 0.695 & \multirow{3}{*}{---- } \\
\hline & & 1.251 & 45.78 & 1736 & 0.581 & \\
\hline & & 1.587 & 54.02 & 2272 & 0.371 & \\
\hline \multirow{3}{*}{$\begin{array}{c}\text { Passive } \\
\text { OFF }\end{array}$} & \multirow{3}{*}{$\begin{array}{l}\text { Modified } \\
\text { Bouc-Wen }\end{array}$} & 0.518 & 20.02 & 999 & 0.518 & \multirow{3}{*}{166.4} \\
\hline & & 0.907 & 34.51 & 1358 & 0.443 & \\
\hline & & 1.191 & 42.79 & 1791 & 0.292 & \\
\hline \multirow{3}{*}{$\begin{array}{c}\text { Passive } \\
\text { ON }\end{array}$} & \multirow{3}{*}{$\begin{array}{c}\text { Modified } \\
\text { Bouc-Wen }\end{array}$} & 0.171 & 7.77 & 613 & 0.171 & \multirow{3}{*}{1048.9} \\
\hline & & 0.423 & 19.36 & 1066 & 0.253 & \\
\hline & & 0.560 & 25.58 & 1366 & 0.208 & \\
\hline \multirow{5}{*}{\multicolumn{2}{|c|}{$\begin{array}{c}\text { FLC } \\
\text { (GA optimization) }\end{array}$}} & 0.197 & 8.51 & 694 & \multirow{5}{*}{$\begin{array}{l}0.197 \\
0.228 \\
0.156\end{array}$} & \multirow{5}{*}{1122.3} \\
\hline & & $(15 \%)$ & $(10 \%)$ & $(13 \%)$ & & \\
\hline & & $\begin{array}{l}0.394 \\
(-7 \%)\end{array}$ & $\begin{array}{l}18.49 \\
(-5 \%)\end{array}$ & $\begin{array}{l}1045 \\
(-2 \%)\end{array}$ & & \\
\hline & & 0.520 & 21.93 & 938 & & \\
\hline & & $(-7 \%)$ & $(-14 \%)$ & $(-31 \%)$ & & \\
\hline
\end{tabular}

Each line represents one DOF $\left(3^{\text {rd }}, 2^{\text {nd }}\right.$ and $1^{\text {st }}$ DOF from top to bottom $)$. Bold values represent the lowest/best solution.

\section{CONCLUSIONS}

A simple optimization procedure based on a genetic algorithm was sucessfully applied to design a FLC for controlling a semi-active MR actuator. Although the proposed fuzzy controller presents a reduced performance in controlling the first floor response, it outperforms the other control modes in reducing peak responses of the third floor, namely the peak acceleration (31\%). Drift responses are also improved compared with the on-damping state (maximum damping force). Thus, the simulation results show that the FLC can reduce the response of the 3-DOFs system using only velocity measurements of the first and third masses with a MR damper at the first mass (i.e., a non-collocated sensor/actuator configuration).

\section{REFERENCES}

[1] Ali, S.F. \& Ramaswamy A. 2008, GA optimized FLC driven semi-active control for phase II smart nonlinear base isolated benchmark building, Structural Control \& Health Monitoring, Vol. 15-5, pp. 797-820.

[2] Braz-César, M. \& Barros, R. 2012, Experimental behaviour and numerical analysis of MR dampers, 15WCEE - 15th World Conference on Earthquake Engineering, Portugal.

[3] Braz-César, M. \& Barros, R. 2017, GA-optimized semiactive fuzzy based controller for seismic vibration reduction of a 3 dof struture with velocity feedback, M2D2017, Portugal.

[4] Byrne, J.P. 2003, GA-optimization of a fuzzy logic controller, Master's Thesis, School of Electronic Engineering, Dublin City University.

[5] Jang, J.R. 1993, ANFIS: Adaptive-network-based fuzzy inference system, IEEE Transactions on Systems, Man and Cybernetics, Vol. 23(3), pp. 665-685.

[6] Spencer Jr., B.F., Dyke, S.J., Sain, M.K., Carlson, J.D. 1997, Phenomenological model of a magnetorheological damper, Journal of Engineering Mechanics, 123, pp. 230.238. 